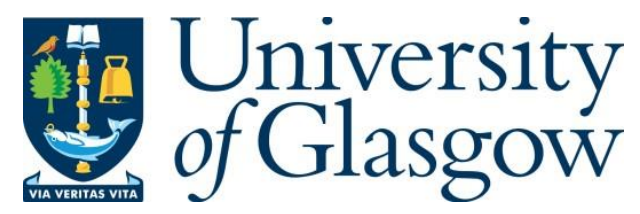

Debnath, S. et al. (2019) Tunable supramolecular gels by varying thermal history. Chemistry: A European Journal, 25(33), pp. 7881-7887

There may be differences between this version and the published version. You are advised to consult the publisher's version if you wish to cite from it.

This is the peer reviewed version of the following article Debnath, S. et al. (2019) Tunable supramolecular gels by varying thermal history. Chemistry: A European Journal, 25(33), pp. 7881-7887, which has been published in final form at http://dx.doi.org/10.1002/chem.201806281

This article may be used for non-commercial purposes in accordance with Wiley Terms and Conditions for Self-Archiving.

http://eprints.gla.ac.uk/183918/

Deposited on: 15 April 2019

Enlighten - Research publications by members of the University of Glasgow http://eprints.gla.ac.uk 


\section{CHEMISTRY A European Journal}

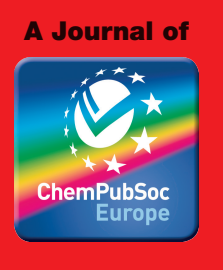

\section{Accepted Article}

Title: Tunable supramolecular gels by varying thermal history

Authors: Sisir Debnath, Sangita Roy, Yousef M Abul-Haija, Pim

Frederix, Susana Ramalhete, Andrew Hirst, Nadeem Javid, Neil Hunt, Sharon Kelly, Jesús Angulo, Yaroslav Khimyak, and Rein Ulijn

This manuscript has been accepted after peer review and appears as an Accepted Article online prior to editing, proofing, and formal publication of the final Version of Record (VoR). This work is currently citable by using the Digital Object Identifier (DOI) given below. The VoR will be published online in Early View as soon as possible and may be different to this Accepted Article as a result of editing. Readers should obtain the VoR from the journal website shown below when it is published to ensure accuracy of information. The authors are responsible for the content of this Accepted Article.

To be cited as: Chem. Eur. J. 10.1002/chem.201806281

Link to VoR: http://dx.doi.org/10.1002/chem.201806281 


\title{
Tunable supramolecular gel properties by varying thermal history
}

\author{
Sisir Debnath, ${ }^{[a],+, \uparrow}$ Sangita Roy, ${ }^{[a], \uparrow}$ Yousef M. Abul-Haija, ${ }^{[a], \S}$ Pim W. J. M. Frederix, ${ }^{[a, b]}$ Susana M. \\ Ramalhete, ${ }^{[\mathrm{c}]}$ Andrew R. Hirst, ${ }^{[\mathrm{a]}, \#}$ Nadeem Javid, ${ }^{[\mathrm{d}]}{ }^{, \|}$Neil T. Hunt, ${ }^{[\mathrm{b}], \#}$ Sharon M. Kelly, ${ }^{[\mathrm{d}]}$ Jesús Angulo, ${ }^{[\mathrm{c}]}$ \\ Yaroslav Z. Khimyak ${ }^{\star[c]}$ and Rein V. Ulijn ${ }^{\star[a, e, f, g]}$
}

\begin{abstract}
We demonstrate that differential pre-heating prior to supramolecular gelation can be used to control the balance between hydrogen bonding and aromatic stacking interactions in supramolecular gels, and consequent systematic regulation of structure and properties. Using a model aromatic peptide amphiphile, Fmoc-tyrosyl-leucine (Fmoc-YL) and a combination of fluorescence, infrared, circular dichroism and NMR spectroscopy, we show that the balance of these interactions can be adjusted by temporary exposure to elevated temperatures in the range 313-365 K, followed by supramolecular locking in the gel state by cooling to room temperature. Distinct regimes can be identified regarding the balance between $\mathrm{H}$-bonding and aromatic stacking interactions, with a transition point at $333 \mathrm{~K}$. Consequently, gels can be obtained with customizable properties, including supramolecular chirality and gel stiffness. The differential supramolecular structures also result in changes in proteolytic stability, highlighting the possibility of obtaining a range of supramolecular architectures from a single molecular structure by simply controlling the pre-assembly
\end{abstract}

[a] Dr. S. Debnath, Dr. S. Roy, Dr. P. W. J. M. Frederix, Dr. Y. M. AbulHaija, Dr. A. Hirst, Dr. N. Javid and Prof. R. V. Ulijn WestCHEM, Department of Pure and Applied Chemistry University of Strathclyde

295 Cathedral Street, Glasgow G1 1XL, UK

E-mail: Rein.Ulijn@asrc.cuny.edu

[b] Dr. P. W. J. M. Frederix and Prof. N. T. Hunt SUPA, Department of Physics University of Strathclyde 107 Rottenrow East, Glasgow G4 0NG, UK

[c] Dr. S. M. Ramalhete, Dr. J. Angulo and Prof. Y. Z. Khimyak School of Pharmacy

University of East Anglia

Norwich Research Park, Norwich NR4 7TJ, UK

E-mail:Y.Khimyak@uea.ac.uk

[d] Dr. S. M. Kelly

Institute of Molecular Cell and Systems Biology

University of Glasgow

Glasgow G12 8QQ, UK

[e] Prof. R. V. Ulijn

Advanced Science Research Center (ASRC) at the Graduate

Center of the City University of New York (CUNY)

85 St Nicholas Terrace, New York 10031, USA

[f] Prof. R. V. Ulijn

Department of Chemistry, Hunter College

City University of New York

695 Park Avenue, New York 10065, USA

[g] Prof. R. V. Ulijn

Ph.D. programs in Biochemistry and Chemistry

The Graduate Center of the City University of New York

New York 10016, USA

Supporting information for this article is given via a link at the end of the document. temperature.

\section{Introduction}

Molecular self-assembly enables the construction of functional supramolecular materials, including gels, with potential applications in biomedicine and nanotechnology. ${ }^{[1]}$ In addition to molecular design of building blocks, which is typically focused on the rational incorporation of molecular features that regulate relative contributions of non-covalent interactions (ionic, $\pi$ - stacking, $\mathrm{H}$-bonding, etc.), it is now widely recognized that the self-assembly process or pathway strongly dictates the supramolecular organization, which in turn dictates function. Indeed, a single molecular structure may give rise to various superstructures. ${ }^{[2]}$ Several approaches have been introduced that enable control of assembly pathways, including the temporary exposure to different solvent compositions, ${ }^{[2 a]}$ varying catalyst concentrations in (bio-) catalytic self-assembly ${ }^{[2 b, c, d]}$ and varying routes to adjusting $\mathrm{pH}^{[2 \mathrm{e}]}$, ionic strength, by using mechanical agitation/sonication ${ }^{[2 \mathrm{~g}, \mathrm{~h}, \mathrm{l}, \mathrm{j}]}$ or temperature. ${ }^{[2 \mathrm{~m}, \mathrm{n}, \mathrm{o}, \mathrm{p}]}$

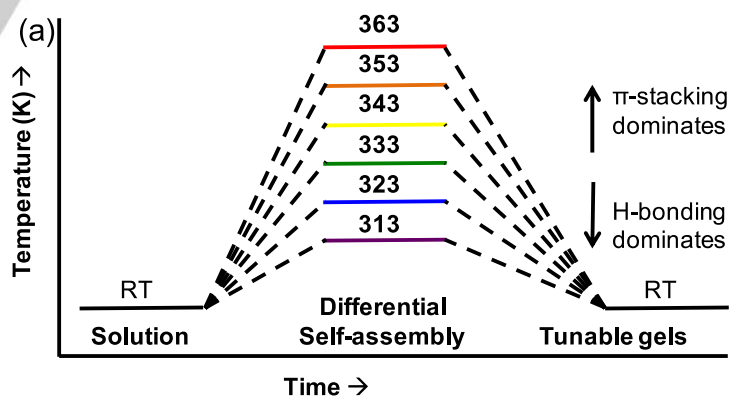

(b)

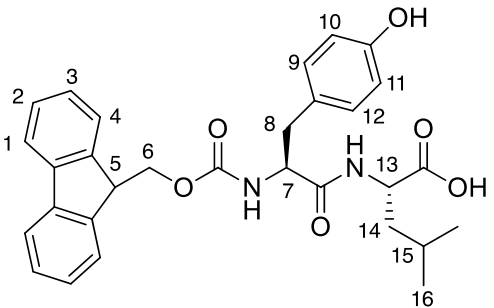

Figure 1. (a) Pathway dependent self-assembly and gelation of an aromatic peptide amphiphile by varying thermal history. Changing assembly temperature and subsequent locking of supramolecular structures by cooling to room temperature (RT), the balance between non-covalent interactions may be regulated, giving rise to tunable gels. (b) Chemical structure of Fmoctyrosyl-leucine (Fmoc-YL); protons numbered for NMR. 
As the strengths of non-covalent interactions that underpin self-assembly (and subsequent gelation) have different temperature dependencies, and by taking advantage of the dramatically reduced molecular mobility upon gelation (achieved by cooling to RT), we reasoned that it should be possible to tune assembly pathways and consequent nanostructure formation by simply varying the thermal history (Figure 1a). We hypothesized that the final structure will be dictated by brief (10 minutes) exposure to elevated temperature prior to gelation at RT. We demonstrate that this thermal memory approach enables control (and inversion) of supramolecular chirality, nanoscale morphology, stiffness and consequent proteolytic stability of gel products.

\section{Results and Discussion}

To demonstrate our thermal memory concept, we use an example of the versatile class of low molecular weight gelators, aromatic peptide amphiphiles, i.e. short peptides functionalized with aromatic moieties. ${ }^{[3]}$ The self-assembly of these structures is dictated both by balancing of $\mathrm{H}$-bonding interactions and hydrophobic aromatic stacking contributions. It has been shown that by chemical design (i.e., by introducing groups that are more or less prone to aromatic stacking), it is possible to control the relative contributions of these interactions, and consequently nanoscale morphology. ${ }^{[4]}$

Self-assembly and gelation of Fmoc-tyrosyl-leucine (Fmoc$\mathrm{YL}$, Figure $1 \mathrm{~b}$ ) was achieved by dissolving $10 \mathrm{mM}$ of Fmoc-YL in $100 \mathrm{mM} \mathrm{pH} 8$ sodium phosphate buffer followed by heating to different temperatures (RT and between $313-363 \mathrm{~K}$ for 10 minutes, see materials and methods) followed by cooling to room temperature to obtain gels (Figure 1a). Transparent gels were formed in each case and their supramolecular organization was analyzed by fluorescence, FTIR, NMR spectroscopy and circular dichroism.

Fmoc-peptides self-assemble through a combination of weak hydrophobic forces, leading to chiral stacking of the fluorenyl groups further stabilized by hydrogen bonding interactions. ${ }^{[3]}$ Fluorescence emission spectra showed two characteristic peaks, a major peak at $320 \mathrm{~nm}$ corresponding to the monomeric Fmoc-YL emission and a broad, low intensity peak at $440-450 \mathrm{~nm}$, corresponding to the formation of excimeric aggregates (Figure 2a). ${ }^{[3 \mathrm{~d}]}$ We observed a progressive quenching of the monomeric emission in the final gel state for samples exposed to increasing pre-gelation temperatures, suggesting that more extensive interactions are present between the fluorenyl groups at elevated $\mathrm{T}$, which may result from more molecules becoming involved in the assembly under these conditions (see NMR results, discussed below).

FT-IR spectroscopy shows that hydrogen bonding between peptidic components is also affected by the pre-gelation temperature. Figure $2 \mathrm{~b}$ shows the FT-IR spectra in the amide I region. In all gels it is possible to observe peaks at $1625 \mathrm{~cm}^{-1}$, which is a characteristic frequency for amide groups in a wellordered $\beta$-sheet type arrangement. In addition, a peak at 1683 $\mathrm{cm}^{-1}$ indicates $\mathrm{H}$-bonding interactions through stacked carbamate carbonyls. ${ }^{[7]}$ It is noted that a weak absorption around $1645 \mathrm{~cm}^{-1}$, which is assigned to less ordered interactions with respect to hydrogen bonding, is present in those gels preexposed to higher temperatures over $333 \mathrm{~K}$ (see the shaded area in Figure $2 b$ ). This increase was observed with a concomitant decrease in the relative intensity of the peak at $1625 \mathrm{~cm}^{-1}$ compared to $1683 \mathrm{~cm}^{-1}$, confirming that the decrease in the number of ordered amide groups (Figure S1). The 1645 $\mathrm{cm}^{-1}$ peak appears at temperatures higher than $333 \mathrm{~K}$, which has been shown previously to be the onset of "melting" of the $\beta$ sheet component in an Fmoc-dileucine hydrogel. ${ }^{[5]}$ This observation supports that less order in $\mathrm{H}$-bonding is induced in the $\beta$-sheet type arrangement by increasing the pre-gelation temperature.
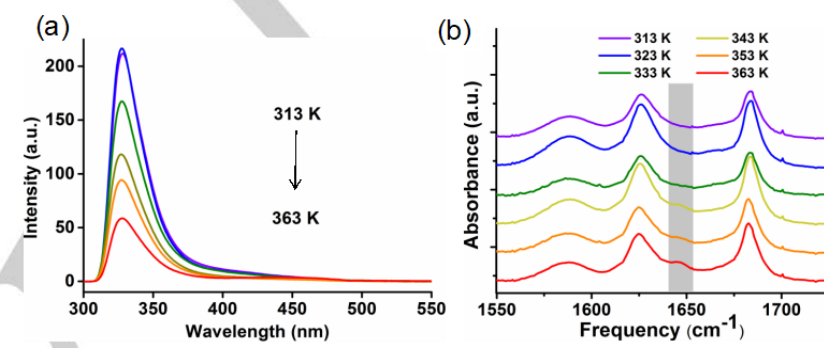

Figure 2. (a) Emission spectra of Fmoc-YL gels $\left(\lambda_{\mathrm{ex}}=280 \mathrm{~nm}\right)$ after temporary heating to different temperatures, followed by cooling to room temperature. (b) FT-IR spectra of the gels, showing the formation of $\beta$-sheet-like arrangements in each case and arising of a weak absorption peak around $1645 \mathrm{~cm}^{-1}$ (shaded area) in samples that were temporary exposed to higher temperature, indicating less ordered $\beta$-sheet type arrangement.

NMR was subsequently used to assess changes in solvent exposure of components of the gelators as a result of thermal history. Solution-state NMR detects mobile molecular components of the gels. H-NMR spectra of Fmoc-YL gels prepared using different pre-assembly temperatures present superimposed sharp and broad peaks with variation in chemical shift values for aromatic (Figure $3 a$ ) and aliphatic protons (Figure S11), which is an indication of the presence of several distinct local environments. This is particularly evident for leucine methyl protons $\left(\mathrm{H}_{16}\right)$, for which four doublets could be detected (Figure S11). These doublets represent magnetically non-equivalent methyl protons from mobile aliphatic chains. These data show that we can detect both free Fmoc-YL molecules in solution, as well as mobile (solvent exposed) moieties of those Fmoc-YL molecules taking part of the supramolecular network. The multiplicity of the detected environments reflects the structural heterogeneities present in the network.

Figure 3a shows that ${ }^{1} \mathrm{H}$ peaks from fluorenyl groups gradually disappear with increasing pre-assembly temperature, presenting severe broadening at $333-343 \mathrm{~K}$. For these samples, the majority of aromatic groups form the core of self-assembled fibers, having their motional freedom significantly reduced, therefore they are not visible by solution-state NMR spectroscopy. ${ }^{[8]}$ Interestingly, this molecular rearrangement 
occurs at the temperature of "melting" of the $\beta$-sheet components, ${ }^{[5]}$ and supports the existence of a more extensive $\pi$-stacked network. As pre-assembly temperature is raised further, fluorenyl aromatic groups display increased peak intensities (Table S1), with appearance of less shielded peaks. This is accompanied by greater mobility of tyrosine aromatic protons, as this residue becomes more solvent exposed. Thus, the rearrangement of the packing of fibers that occurs upon gelation from higher temperature solution has an effect on the organization of the fiber/solution interfaces and it is reflected by the appearance of both aromatic and aliphatic regions on the spectra, suggesting that the peptide side chains become more mobile, exposing the tyrosine and leucine side chains, and reducing ordered $\mathrm{H}$-bonding.

Next, the impact of thermal history on supramolecular chirality was tested, using circular dichroism. The extensive interactions between fluorenyl groups result in intense $C D$ signals. ${ }^{[2]}$ Focusing on the contribution of induced supramolecular chiral organization of the (achiral) fluorenyl moiety at $305 \mathrm{~nm}$, we observe that the absolute polarization is strongly dependent on the pre-gelation self-assembly temperature (Figure 3c). Previously, using sub-gelation concentrations, we observed that Fmoc-YL demonstrates a positive CD peak at $\sim 305 \mathrm{~nm}$, in addition to strong shorter wavelength signals centered around $275 \mathrm{~nm}$ which may include a contribution from the tyrosine residues ${ }^{[9]}$ Upon increasing pre-gelation temperatures, positive peaks in the 275 and 305 regions invert to a negative signal (Figure $3 c$ ) with the inversion point coinciding with the lowest relative mobility of fluorenyl and tyrosine residues observed by NMR (333-343K). Additionally, CD spectra of pre-gelation solutions at elevated temperatures (Figure S4) show that Fmoc$\mathrm{YL}$ solutions have a negative $C D$ signal at relatively low temperatures $(313-333 \mathrm{~K})$ with intensity reduced at elevated temperatures $(343 \mathrm{~K}$ and $353 \mathrm{~K}$ ). These data show that the chiral inversion point indeed at $333 \mathrm{~K}$ reflects the point where structure remains at elevated temperature, however the $C D$ signals invert upon cooling and gelation. It is to be noted here that no significant linear dichroism contribution was found at any temperature (Figure S5). These observations indicate that the formation of supramolecular assemblies with (preferential) opposite handedness can be controlled by the pre-assembly temperature, overriding the configuration of the molecular chiral center. The changes observed are consistent with a less ordered $\mathrm{H}$-bonded network present above $333 \mathrm{~K}$, in line with the increased intensity of the IR absorption peak at $1645 \mathrm{~cm}^{-1}$ (Figure 2b).

The aliphatic region of Fmoc-YL displays intense resonances in solution-state NMR throughout the range of temperatures (Figure S11) and that peak intensities and line widths for leucine protons are maintained relatively constant (Figure S12b). These observations suggest that these aliphatic residues remain solvent exposed even after rearrangement of the aromatic residues. Consequently, the ratio between aromatic and aliphatic peak intensities is affected by the pre-assembly temperature (Tables S1 and S2). This ratio is consistently low (ca. 0.10$)$, showing that most of fluorenyl and tyrosine residues are deeply inserted into the gel fibers. Overall, lower peak intensities are detected for all ${ }^{1} \mathrm{H}$ peaks as the temperature of assembly is raised (Table S1) due to a greater amount of gelator molecules incorporated into the gel fibers, which is in good agreement with the decreased monomeric emission recorded at higher pre-gelation temperatures (320 nm peak in Figure 2a).

Saturation transfer difference (STD) NMR experiments enable the mode of interaction of molecules with the interfaces of supramolecular gels to be studied. ${ }^{[10]}$ Briefly, in STD NMR we selectively induce cancellation of proton intensities of the network (saturation) by a selective low power irradiation at a frequency where only broad resonances from the rigid structures are encountered. Those Fmoc-YL molecules exchanging between the gel fibers and the isotropic pools of solvent will carry part of that saturation to the solution "free" state, where we can detect the reduction in the intensities of the Fmoc-YL protons as a signal in a difference spectrum (see Materials and Methods). As a result, Fmoc-YL protons that were in close proximity to the surface of the fibers in the "bound" state will show stronger STD intensities, in comparison to those more solvent-exposed, depicting the mode of interaction of the Fmocdipeptide within the gel network. The saturation maps from figure $3 \mathrm{~b}$ show that the saturation transferred from the gel network to the aromatic peaks is increases when comparing gels self-assembled from temporary exposure to 313 and $333 \mathrm{~K}$, respectively, but it is significantly reduced in those pre-exposed to $363 \mathrm{~K}$.
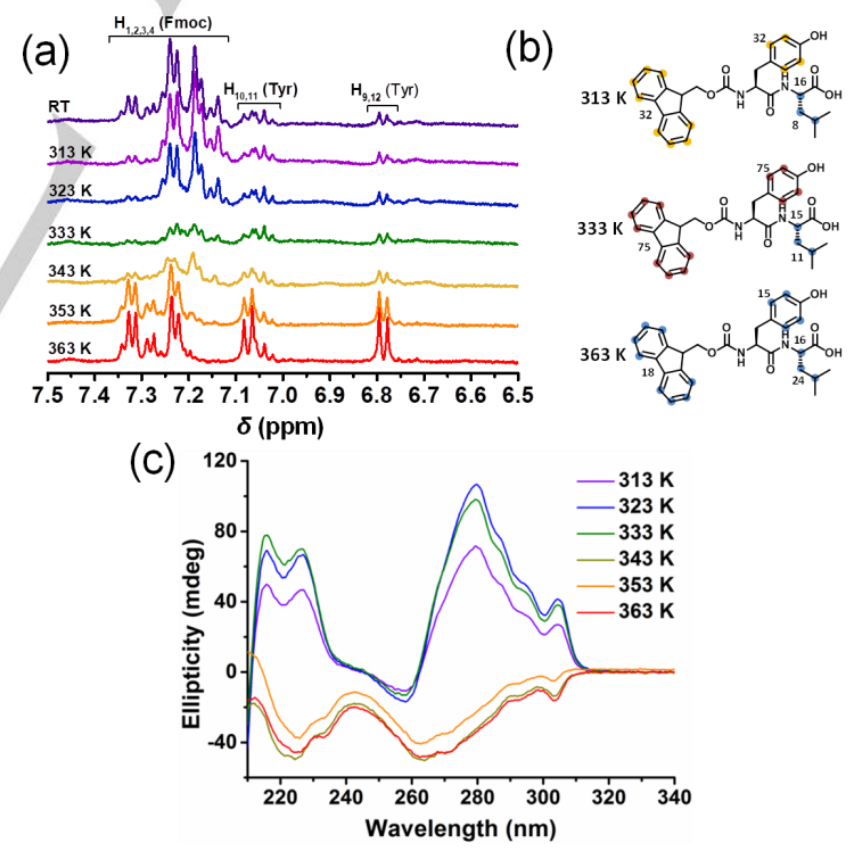

Figure 3. (a) Aromatic region of ${ }^{1} \mathrm{H}-\mathrm{NMR}$ spectra of gel samples prepared using different pre-assembly temperatures. (b) Saturation maps (STD initial slopes, $\mathrm{STD}_{0}$ ) of Fmoc-YL gels prepared at 313,333 and $363 \mathrm{~K}$, with legend of the color scheme (strong (red): 70-100 s-1, medium (yellow): 30-69 $\mathrm{s}^{-1}$, weak (blue): $0-29 \mathrm{~s}^{-1}$ ). Analysis of methyl groups at the side chain of leucine $\left(\mathrm{H}_{16}\right)$ is described in the supporting information (Table S3). (c) CD spectra of Fmoc-YL hydrogels assembled at different temperatures and 
subsequently locked by gelation at room temperature, showing chiral inversion Corresponding HT data is shown in Figure S2.

Interestingly, as in the NMR spectra of these gels both types of signals can be observed, i.e. from Fmoc-YL molecules in the isotropic pools of solvent and from the mobile moieties of the network, it is possible to detect STD signals arising from intranetwork transfer of saturation (i.e. spin diffusion). Intermolecular STDs provided indirect evidence of the changes occurring at the fiber surfaces (due to different orientations of the exchanging gelator molecule in the state bound to the network). In contrast, intra-network STDs detected on the aliphatic protons clearly show that the saturation transferred from the network to leucine protons is very sensitive to thermal history (Table S3). These phenomena are discussed in more detail in the supporting information. This provides direct proof of changes in the internal structure of the network due to the different pre-gelation temperatures. Altogether, these variations reflect different modes of interaction of the exchanging gelator molecules with the surface of the fibers, and support that there are significant structural modifications at the interfaces due to reorganization of the core of gel fibers. In summary, FTIR and fluorescence emission spectroscopies proved predominance of a certain type of supramolecular interaction (H-bonding or $\pi$-stacking), and NMR spectroscopy indicated changes in the packing modes of gelator molecules treated at different temperatures.

The three regimes of fiber organization identified during NMR studies are consistent with CD studies. The modification of local environments detected by NMR spectroscopy correlate with the chiral inversion identified by $C D$ - the marked differences in mobility of protons show a mode of packing at lower temperatures in which tyrosine is less mobile, whereas the reorganization of the fibers imposed by the chirality switch at higher temperatures leads to more solvent exposed tyrosine residues as they become less integrated through reduction of $\mathrm{H}$ bonding.

While Bing Xu's group previously reported the expected, opposite circular dichroism (CD) for gels of Fmoc-dialanine, dictated by the stereochemistry (either $D$ or $L$ ) of the building blocks when identical gelation routes were used for each stereoisomer, ${ }^{[11]}$ chiral inversion of Fmoc-peptide assemblies has been observed previously when comparing enzymatic to non-enzymatic formation of chiral nanostructures ${ }^{[2 \mathrm{c}]}$ and upon co-assembly with proteins, ${ }^{[12]}$ clearly showing that subtle changes in interactions can cause these fluorenyl groups rearrange. It should be noted that previous reports showed that optical activity of polythiophene-based films and cholesterolbased organo-gels could be switched between two states of chirality by adjusting the cooling time. ${ }^{[13]}$ Our data clearly show that chirality may be controlled and even inverted purely by route dependent balancing of non-covalent interactions.

The mechanical properties of the gels were measured using oscillatory rheology. For all gels, the storage modulus $(G)$ is an order of magnitude higher than the loss modulus ( $G^{\prime \prime}$ ) indicating that they are viscoelastic in nature and behave as typical gels. Hydrogels formed by high pre-assembly

temperatures are mechanically stiffer compared to those formed at low temperatures (Figure S6), with elastic moduli increasing from around 0.3 to $1 \mathrm{kPa}$. Also, the temperature at which the gel melted and showed gravitational flow $\left(T_{\text {gel }}\right)$ was found to increase with increase in the pre-assembly temperature of the gels (Figure S6). These results suggest that the strength of the gel is proportional to the pre-assembly temperature and that it is associated with the changes in concentration of molecules involved in fiber formation, reflected by reduced ${ }^{1} \mathrm{H}-\mathrm{NMR}$ peak intensities (Figure S12a). This can be correlated further with the morphological network properties of the gels as observed by atomic force microscopy. Dried films of the gels formed at low temperatures yield shorter and wider fibers (Figure 4a).

The structures formed clearly represent different assembled states, which are kinetically trapped due to the reduced mobility in the gel phase. In order to assess which of the self-assembling structures ( $\mathrm{H}$-bonding or $\pi$-stacking dominated) represents a lower energy structure, we subsequently controlled the cool time after self-assembly at elevated temperature, from 10 to $300 \mathrm{~min}$, thus allowing structural reorganization towards a thermodynamically more optimized structure. We observe preferential formation of the $\pi$-stacking dominated structure having negative Cotton effect at $305 \mathrm{~nm}$ for the slow cooled gels, irrespective of the temperature they were heated to (Figure S3).

(a)
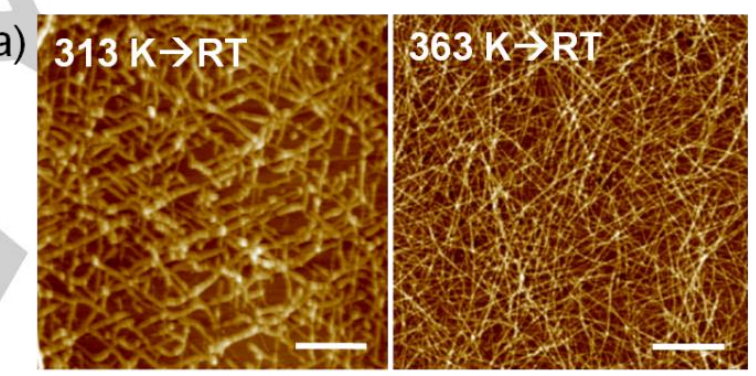

(b)

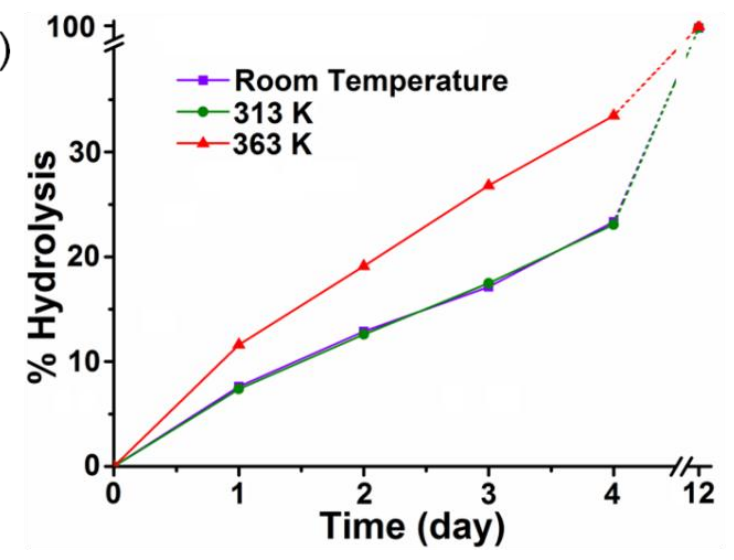

Figure 4. (a) AFM images of the Fmoc-YL gels formed by varying thermal history through temporal exposure to $313 \mathrm{~K}$ and $363 \mathrm{~K}$ (scale bar=2 $\mu \mathrm{m})$. (b) Differential hydrolysis of Fmoc-YL gels formed at different temperature by proteinase $\mathrm{K}$.

Finally, we investigate whether the induced helicity and differential self-assembly of these Fmoc-peptide nanostructures 
has an impact on their susceptibility to proteolytic degradation. Proteinase $\mathrm{K}$, a non-selective protease used previously for the digestion of Phe-Phe nanotubes, ${ }^{[14]}$ shows enhanced degradation rate for the nanofibers that are dominated by aromatic interactions (assembled at $363 \mathrm{~K}$ ), compared to those that have a comparatively higher $\beta$-sheet content (assembled at RT or $313 \mathrm{~K}$, Figures $4 \mathrm{~b}$ and S6-S8). Upon longer exposure (12 days) all the gels are eventually degraded completely. Thus, there is a remarkable inverse relationship between thermodynamic stability (m-stacking dominated) and proteolytic stability ( $\beta$-sheet dominated) for these systems. We note that there may exist an analogy with the proteolytic resistance observed in the cross-beta structures present in amyloid fibers. ${ }^{[15]}$ Our approach provides new opportunities for enhancing proteolytic stability of biomaterials by processing, while retaining the naturally occurring, all $L$ - configuration.

\section{Conclusions}

In summary, the results show that a single molecular structure gives rise to a variety of supramolecular architectures using kinetically controlled self-assembly by simply varying the thermal history. Thermal tuning of supramolecular interactions, followed by locking structures by gelation, provides a powerful route to tailor gel properties. Remarkably, the self-assembly route can be used to direct and even invert the helical bias of the supramolecular chirality of a dipeptide gelator by rebalancing $\mathrm{H}$ bonding and $\pi$-stacking interactions. This control is achieved by directing the supramolecular structures towards different local minima through temperature-dependent pre-assembly. At high temperatures, aromatic interactions dominate with less order in $\mathrm{H}$-bonded arrangements, while assembly at lower temperatures gives rise to systems dominated by a more ordered hydrogen bonding network. This structural switch induced by pre-assembly temperature was probed locally by NMR spectroscopy and at a larger length scale by $C D$ spectroscopy. Consequently, the different balance of interactions influenced the proteolytic degradation of the material, with lower degradation rates observed for the systems that had a more ordered $\mathrm{H}$-bonding structure. Such tuning of the properties of supramolecular hydrogels provides ways to achieve control over fabrication of these functional nanomaterials and further confirms that design rules should include both thermodynamic and kinetic considerations.

\section{Experimental Section}

Non-equilibrium assembly and hydrogelation: $5.16 \mathrm{mg}$ of $\mathrm{N}$ (fluorenyl-9-methoxycarbonyl) (Fmoc)-YL (10 $\left.\mathrm{mmol} \mathrm{L}^{-1}\right)$, was dispersed in a $1 \mathrm{~mL}$ volume of $100 \mathrm{mM} \mathrm{pH} 8$ sodium phosphate buffer. Alternate vortexing and sonication for 5 minutes resulted in the complete dissolution of Fmoc-YL, which self-assembled to form a self-supporting gel in 2-3 hours. The resulting gels were left overnight followed by heating to different temperatures for 10 minutes in an oil bath, which were then allowed to cool gradually in the same oil bath to room temperature. Gels were again left to stabilize overnight before measurements were performed.

Fluorescence spectroscopy: Fluorescence emission spectra were measured on a Jasco FP-6500 spectrofluorometer with light measured orthogonally to the excitation light, at a scanning speed of $100 \mathrm{~nm} \mathrm{~min}{ }^{-1}$. The excitation wavelength was $280 \mathrm{~nm}$, and emission data were recorded in the range between 300 and $550 \mathrm{~nm}$. The spectra were measured with a bandwidth of $5 \mathrm{~nm}$ with a medium response and a $1 \mathrm{~nm}$ data pitch. Quartz cells with a path length of $10 \mathrm{~mm}$ were used for the study.

Circular dichroism and linear dichroism (CD and LD): Spectra were measured on a Jasco J-800 spectropolarimeter with a demountable cell of path length of $0.001 \mathrm{~cm}$. Temperature dependent CD was measured with a Jasco J-600 spectropolarimeter with Quartz cells of $0.002 \mathrm{~cm}$ path length.

Atomic force microscopy (AFM): AFM measurements were performed using a Veeco Multi Mode with NanoScope IIID Controller Scanning Probe Microscope. After the preparation of the Fmoc-YL gel by aforementioned protocol, part of the gel was diluted 5 times with double distilled water and cast $10 \mu \mathrm{L}$ of diluted sample on the mica plate and dried overnight before imaging.

FT-IR spectroscopy: spectra were recorded on a Bruker optics Vertex 70 spectrophotometer. All hydrogels were prepared in the same way as described earlier in $100 \mathrm{mM}$ pH 8 sodium phosphate buffer in $\mathrm{D}_{2} \mathrm{O}$ instead of $\mathrm{H}_{2} \mathrm{O}$. The hydrogels were loaded between two $\mathrm{CaF}_{2}$ windows with a $25 \mathrm{~mm}$ spacer. The spectra were taken in the region between 800 and $4000 \mathrm{~cm}^{-1}$ with a resolution of $1 \mathrm{~cm}^{-1}$ and averaged over 25 scans. Spectra were background subtracted to correct for atmospheric interference. Spectra from $\mathrm{D}_{2} \mathrm{O}$ without peptide were subtracted from the results. Spectra have been vertically offset for clarity.

NMR spectroscopy: Solution-state NMR experiments were performed at $298 \mathrm{~K}$ using a Bruker Avance I spectrometer operating at ${ }^{1} \mathrm{H}$ frequency of 499.69 MHz equipped with a $5 \mathrm{~mm}$ probe. Hot solutions were transferred into NMR tubes and allowed to gelate inside the tube. ${ }^{1} \mathrm{H}-\mathrm{NMR}$ spectra were acquired using excitation sculpting for water suppression with a recycle delay of $4 \mathrm{~s}$ and 1024 scans. STD NMR experiments ${ }^{[6 a]}$ were performed with selective saturation $\left(S_{T D_{0}}\right)$ at $0 \mathrm{ppm}$ by a train of Gaussian pulses, each one with duration of $50 \mathrm{~ms}$ and WATERGATE for solvent suppression (performed using the pulse sequence stddiffgp19.2), acquired with a total of 256 scans. STD spectra were created by the subtraction of the on-resonance spectrum $\left(S T D_{\text {on }}\right)$ to an off-resonance spectrum $\left(S T D_{\text {off }}\right.$ ), acquired with no selective saturation (40 ppm).

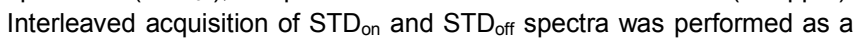
pseudo-2D experiment to minimize artifacts caused by variations throughout the experiment. Each pair of experiments was acquired at variable saturation times ranging from 0.25 to $6 \mathrm{~s}$, varying the relaxation delay inversely to keep the total experimental time per scan constant. Signal intensity in the difference spectrum relative to the signal intensity in the $S T D_{\text {off }}$ spectrum was used to determine the fractional STD response. Since all fluorenyl aromatic peaks showed the same variation, we used a general STD integration for these aromatics. STD build-up curves were fitted mathematically to the mono-exponential function $\operatorname{STD}_{\text {(tsat) }}=\operatorname{STD}^{\max }\left(1-\mathrm{e}^{\left(-\mathrm{k}_{\text {sat }} \cdot \mathrm{t}_{\text {sat }}\right)}\right)$, where $\operatorname{STD}^{\max }$ is the maximum possible STD at very long saturation times, $\mathrm{k}_{\text {sat }}$ is the saturation rate constant and $t_{\text {sat }}$ is the saturation time. Initial slope values of the build-up curves, STD 0 , were obtained from the product STD ${ }^{\max } \times \mathrm{k}_{\mathrm{sat}} \cdot{ }^{[6 \mathrm{~b}]}$ 
Gel-to-sol transition temperature ( $\left.T_{\text {gel }}\right)$ : The gel-to-sol transition temperature $\left(T_{\text {gel }}\right)$ was determined by placing the hydrogels (prepared at different temperatures as stated earlier) containing screw-capped glass vial (internal diameter $10 \mathrm{~mm}$ ) into a thermostat oil bath and raising the temperature $1 \mathrm{~K} \mathrm{~min}^{-1}$. With each $1 \mathrm{~K}$ increase, the gel-sol transition was checked by inversion of the vials. The $T_{\text {gel }}$ was defined as the temperature at which the gel melted and showed gravitational flow. The macroscopic gel-sol transitions were also confirmed by temperature dependent CD spectroscopy.

Proteolytic degradation: Fmoc-YL (11.11 mM) gels were prepared (900 $\mu \mathrm{L}$ each) by aforementioned protocol (one at room temperature and other two by heating at 313 and $363 \mathrm{~K}$ ). Then $100 \mu \mathrm{L}$ of $10 \mathrm{mg} \mathrm{mL}^{-1}$ proteinase $\mathrm{K}$ were mixed in each sample and vortexed for 1 minute. The final concentrations of gels were $10 \mathrm{mM}$ and of enzyme was $1 \mathrm{mg} \mathrm{mL}^{-1}$. Afterwards, every $24 \mathrm{~h}$ up to 12 days samples were prepared for HPLC analysis by dissolving $20 \mu \mathrm{L}$ of the gel in the $50: 50 \mathrm{H}_{2} \mathrm{O}: \mathrm{ACN}$ solution. Degradation of Fmoc-YL was analyzed by HPLC.

High-Performance Liquid Chromatography (HPLC): A Dionex P680 HPLC pump was used to quantify conversions of the proteolytic degradation reaction. A $20 \mu \mathrm{L}$ sample was injected onto a MachereyNagel $\mathrm{C}_{18}$ column of $250 \mathrm{~mm}$ length with an internal diameter of $4.6 \mathrm{~mm}$ and $5 \mu \mathrm{m}$ fused silica particles at a flow rate of $1 \mathrm{~mL} \mathrm{~min}^{-1}$ [eluting solvent system: linear gradient of $20 \%(\mathrm{v} / \mathrm{v})$ acetonitrile in water for $4 \mathrm{~min}$ and gradually rising to $80 \%(\mathrm{v} / \mathrm{v})$ acetonitrile in water at $35 \mathrm{~min}$; this concentration was kept constant until $40 \mathrm{~min}$ when the gradient was decreased to $20 \%(\mathrm{v} / \mathrm{v})$ acetonitrile in water at $42 \mathrm{~min}]$. The sample preparation involved mixing $50 \mu \mathrm{L}$ of gel with acetonitrile-water $(950 \mu \mathrm{L}$, 50:50 mixture) containing $0.1 \%$ trifluoroacetic acid. The purity of each identified peak was determined by the ultraviolet (UV) detection at 280 $\mathrm{nm}$.

Oscillatory rheology: To verify the mechanical properties of the resulting hydrogels, dynamic frequency sweep experiments were carried out on a strain-controlled rheometer (Kinexus Pro rheometer) using parallel-plate geometry $(20 \mathrm{~mm}$ diameter). The experiments were performed at $298 \mathrm{~K}$, and this temperature was controlled throughout the experiment using an integrated electrical heater. Additional precautions were taken to minimize solvent evaporation and to keep the sample hydrated: a solvent trap was used, and the internal atmosphere was kept saturated. To ensure that the measurements were made in the linear viscoelastic regime, an amplitude sweep was performed, and the results showed no variation in elastic modulus $\left(G^{\prime}\right)$ and viscous modulus $\left(G^{\prime \prime}\right)$ up to a strain of $1 \%$. The dynamic modulus of the hydrogel was measured as a frequency function, where the frequency sweeps were carried out between 0.1 and $100 \mathrm{~Hz}$. The gels were made in small fractions in widemouth vials from which they were transferred with a spatula for rheological measurements. The measurements were repeated 3 times to ensure reproducibility, with the average data shown.

\section{Author Information}

\section{Current Addresses}

‡ Department of Chemistry, Serampore College, 9, William Carey Sarani, Serampore, Hooghly, West Bengal, India, Pin-712201.

$\S$ WestCHEM, School of Chemistry, University of Glasgow, Glasgow G12 $8 Q Q$, UK.
\| School of Chemistry and Biosciences, University of Bradford, Bradford BD7 1DP, UK.

\# Department of Chemistry, University of York, York Y010 5DD, UK.

\section{Author Contributions}

RVU conceived the idea and managed the research team. ARH designed the thermal history experimental protocols and was responsible for early stage characterization of the gels. SD, SR, $\mathrm{NJ}$, YMA-H were responsible for synthesis and characterization, fluorescence spectroscopy, proteolysis, HPLC, AFM. PWJMF was responsible for FTIR. NTH helped with interpretation of FTIR. NJ, YMA-H was responsible for CD, SMK helped with interpretation. SMR performed NMR experiments and data interpretation was guided by JA and YZK. SD, SR, PWJMF, SMR, YMA-H, ARH, YZK and RVU co-wrote the paper with input from all the authors. † These authors contributed equally.

\section{Acknowledgements}

The research leading to these results has received funding from the European Research Council under the European Union's Seventh Framework Programme (FP7/20072013)/EMERgE/ERC Grant Agreement No. (258775). (U.K.) We are grateful for financial support from the University of East Anglia through fully funded Ph.D. studentship for SMR. We thank Prof. Alvaro Mata (QMUL) for helpful discussions.

Keywords: self-assembly pathway, peptide amphiphiles, hydrogel, supramolecular chemistry, thermal history

[1] a) M. J. Webber, E. A. Appel, E. W. Meijer, R. Langer, Nat. Mater. 2016 15, 13-26; b) T. Aida, E. W. Meijer, S. I. Stupp, Science 2012, 335, 813817; c) S. Zhang, Nat. Biotechnol. 2003, 21, 1171-1178; d) D.B. Amabilino, D.K. Smith, J. W. Steed, Chem. Soc. Rev. 2017, 46, 24042420; e) E.R. Draper, D.J. Adams, D. J. Chem 2017 3, 390-410.

[2] a) Y. Tidhar, H. Weissman, S. G. Wolf, A. Gulino, B. Rybt-chinski, Chem. Eur. J., 2011, 17, 6068-6075; b) R. Haburcak, J. F. Shi, X. W. Du, D. Yuan, B. Xu, J. Am. Chem. Soc. 2016, 138, 15397-15404; c) A. R. Hirst, S. Roy, M. Arora, A. K. Das, N. Hodson, P. Murray, S. Marshall, N. Javid, J. Sefcik, J. Boekhoven, J. H. van Esch, S. Santabarbara, R. V. Ulijn, Nat. Chem. 2010, 2, 1089-1094; d) J. Boekhoven, J. M. Poolman, C. Maity, F. Li, L. Van der Mee, C. B. Minkenberg, E. Mendes, J. H. Van Esch, R. Eelkema, Nat. Chem., 2013, 5, 433-437; e) J. Raeburn, A. Z. Cardoso, D. J. Adams, Chem. Soc. Rev. 2013, 42, 5143-5156; f) P. A. Korevaar, C. J. Newcomb, E. W. Meijer, S. I. Stupp, J. Am. Chem. Soc, 2014, 136, 8540-8543; g) T. Tantakitti, J. Boekhoven, X. Wang, R. Kazantsev, T. Yu, J. Li, E. Zhuang, F. Zandi, J. Ortony, C. Newcomb, L. Palmer, S. Gajendra, M. de la Cruz, G. Schatz, S. Stupp, Nat. Mater. 2016, 15, 469-476; h) E. R. Draper, H. Su, C. Brasnett, R. J. Poole, S. Rogers, H. Cui, A. Seddon, D. J. Adams, Angew. Chem. Int. Ed. 2017, 56, 10467-10470; i) T. Fukui, S. Kawai, S. Fujinuma, Y. Matsushita, T. Yasuda, T. Sakurai, S. Seki, M. Takeuchi, K. Sugiyasu, Nat. Chem. 2017, 9, 493-499; j) W. Helen, P. de Leonardis, R.V. Ulijn, J. Gough and N. Tirelli, Soft Matter, 2011, 7, 
1732-1740; k) F. Rodrı'guez-Llansola, J. F. Miravet, B. Escuder, Chem. Commun. 2009, 0, 209-211; I) P. Duan, H. Cao, L. Zhang, M. Liu, Soft Matter, 2014, 10, 5428-5448; m) Z. Pang, Y. Wei, N. Wang, J. Zhang, Y Gao, S. Qian, Int. J. App. Pharm. 2018, 548, 625-635. n) I. A. Coates, D. K. Smith, Chem. Eur. J. 2009, 15, 6340-6344; o) V. J. Nebot, S. Diaz-Oltra, J. Smets, S. F. Prieto, J. F. Miravet, B. Escuder, Chem. Eur. J. 2014, 20, 5762-5767; p) J. Cui, A. Liu, Y. Guan, J. Zheng, Z. Shen $X$. Wan, Langmuir 2010, 26, 3615-3622.

[3] a) K. Tao, A. Levin, L. Adler-Abramovich, E. Gazit, Chem. Soc. Rev., 2016, 45, 3935; b) X. Du, J. Zhou, J. Shi and B. Xu, Chem. Rev. 2015 115, 13165-3953; c) S. Fleming, R. V. Ulijn, Chem. Soc. Rev. 2014, 43, 8150-8177.

[4] a) C. G. Pappas, Y. M. Abul-Haija, A. Flack, P. W. J. M. Frederix, R. V. Ulijn Chem. Commun. 2014, 50, 10630-10633; b) M. Hughes, P. W. J. M. Frederix, J. Raeburn, L. S. Birchall, J. Sadownik, F. C. Coomer, I. H. Lin, E. J. Cussen, N. T. Hunt, T. Tuttle, S. J. Webb, D. J. Adams, R. V. Ulijn, Soft Matter 2012, 8, 5595-5602; c) D. M. Ryan, T. M. Doran, S. B. Anderson and B. L. Nilsson, Langmuir 2011, 27, 4029-4039; d) D. M. Ryan, S. B. Anderson and B. L. Nilsson, Soft Matter 2010, 6, 3220 3231; e) Y. M. Abul-Haija, G. G. Scott, J. K. Sahoo, T. Tuttle, R. V. Ulijn, Chem. Commun. 2017, 53, 9562-9565.

[5] P. W. J. M. Frederix, R. Kania, J. A. Wright, D. A. Lamprou, R. V. Ulijn, C. J. Pickett, N. T. Hunt, Dalton Trans. 2012, 41, 13112-13119.

[6] a) M. Mayer, B. Meyer, Angew. Chem. Int. Ed. 1999, 38, 1784 b) J. Angulo, P. M. Enríquez - Navas, P. M. Nieto, Chem. Eur. J. 2010, 16, 7803-7812.
[7] S. Fleming, P. W. J. M. Frederix, I. R. Sasselli, N. T. Hunt, R. V. Ulijn, T. Tuttle, Langmuir 2013, 29, 9510-9515.

[8] a) M. Wallace, J. A. Iggoa, D. J. Adams, Soft Matter 2015, 11, 7739 7747 ; b) B. Escuder, M. LLusar, J. F. Miravet, J. Org. Chem. 2006, 71, 7747-7752.

[9] S. Fleming, S. Debnath, P. W. J. M. Frederix, N. T. Hunt, R. V. Ulijn, Biomacromolecules, 2014, 15, 1171-1184.

[10] S. M. Ramalhete, K. P. Nartowski, N. Sarathchandra, J. S. Foster, A. N Round, J. Angulo, G. O. Lloyd, Y. Z. Khimyak, Chem. Eur. J. 2017, 23, 8014-8024.

[11] Y. Zhang, H. Gu, Z. Yang, B. Xu, J. Am. Chem. Soc., 2003, 125, 13680-13681.

[12] N. Javid, S. Roy, M. Zelzer, Z. Yang, J. Sefcik, R. V. Ulijn, Biomacromolecules 2013, 14, 4368-4376.

[13] a) M. M. Bouman and E. W. Meijer, Adv. Mater., 1995, 7, 385-387; b) K. Murata, M. Aoki, T. Suzuki, T. Harada, H. Kawabata, T. Komori, F. Ohseto, K. Ueda, S. J. Shinkai, J. Am. Chem. Soc., 1994, 116, 66646676 .

[14] M. Reches, E. Gazit, Science, 2003, 300, 625-627.

[15] D. M. Hartley, C. Zhao, A. C. Speier, G. A. Woodard, S. Li, Z. Li, T. J. Walz, J. Biol. Chem., 2008, 283, 16790-16800. 


\section{FULL PAPER}

Self-assembly history matters: Thermal history can be used as a simple route of controlling structure and function in supramolecular gels. Changing assembly temperature of a model aromatic peptide amphiphile and subsequent locking of supramolecular structures by cooling gives rise to tunable gels. The balance between hydrogen bonding and hydrophobic interactions may be regulated, which dictates nanofiber formation. It is therefore possible to obtain a range of supramolecular architectures from a single molecular structure.
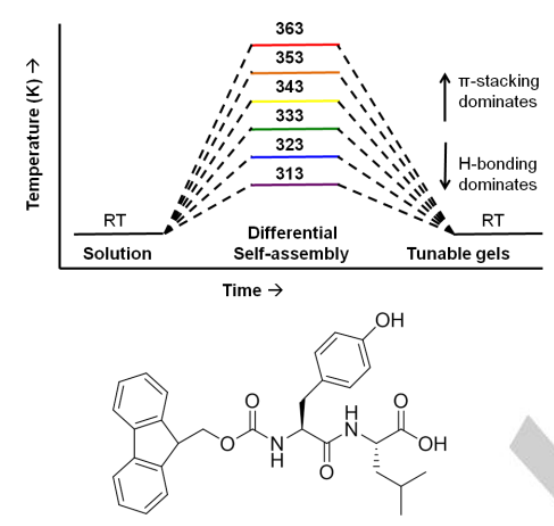

S. Debnath, S. Roy, P. W. J. M. Frederix, S. M. Ramalhete, Y. M. Abul-Haija, A. R. Hirst, N. Javid, N. T. Hunt, S. Kelly, J. Angulo, Y. Z. Khimyak* and R. V. Ulijn*

Page No. - Page No.

Tunable supramolecular gels by varying thermal history 